\title{
ON THE APPLICATION OF A NUMERICAL ALGORITHM FOR HOPF BIFURCATION TO THE HUNTING OF A WHEELSET
}

\author{
R. R. HUILGOL, R. JANUS, M. A. LOHE and T. W. SAG'
}

(Received 5 July 1982; revised 6 January 1983)

\begin{abstract}
The main ideas of Hopf bifurcation theory and its relevance to the development of periodic motions of an autonomous system depending on a parameter are presented, and an algorithm for the computation of the orbits is described. It is then shown that a model system for the motion of a wheelset can be cast in the form amenable to Hopf bifurcation theory. Numerical results for the period and amplitudes of the lateral and yaw motions are obtained in terms of the forward speed of the wheelset, and the wheel-rail profile parameters

It is found that the period of oscillation decreases while the lateral and yaw motion amplitudes increase as the forward speed increases, for any given rail and wheel profile. While the effect of wheel curvature on the lateral motions seems to be non-existent, its effect on the yaw motion amplitude and the period is to increase them very slightly as the wheel profile changes from a conical to a curved profile. On the other hand, the effect of rail curvature on the lateral amplitude, for instance, is significant; the larger the curvature the smaller the amplitude for a given forward speed.
\end{abstract}

\section{Introduction}

About 60 years ago, Carter laid the foundations of the subject of hunting instabilities in trains; a full list of his works is given in the survey article by Law and Cooperrider [24]. Although much work has been done, especially in the last twenty five years, a comprehensive knowledge of the dynamic behaviour of trains and even that of the wheelset-the simplest component-eludes us still. The main reason is that the equations which govern the motions of the above are nonlinear. The nonlinearities are of two kinds: those that are analytic functions of the lateral displacements, the rolling, yawing and pitching motions and the wheel

\footnotetext{
${ }^{1}$ School of Mathematical Sciences, Flinders University of South Australia, Bedford Park, S.A. 5042.

(C) Copyright Australian Mathematical Society 1984, Serial-fee code 0334-2700/84
} 
and rail profiles and their interacting forces; and, secondly, those that are nonanalytic functions, such as flange-rail contact forces which are akin to Heaviside functions. Usually, a compromise between the two sets of nonlinearities is arrived at when a mathematical model of the motion of a train or a wheelset is derived. Using this latter approach, De Pater [10] Brann [3], Cooperrider [9], Law and Brand [23], Wickens [32,33] and others have obtained numerical and analytical solutions to the problems of a single wheelset or a truck. For a survey up to 1974, see [24], and for more recent work, see De Pater [11].

In the present paper, we wish to explore the utility of a method which is different from that used so far. The method relies on using the forward speed $V$ as a parameter, because once the design parameters are fixed, the forward speed $V$ is the only variable (parameter) in the equations of motion. The latter being nonlinear, the solution to the equations may be periodic, quasi-periodic or aperiodic functions of time depending on $V$ as a parameter.

The simplest solution of the three is the case of self-sustained oscillations, i.e., periodic motions. It is our aim in this paper to examine this matter thoroughly for a simple model, which is an extension of that of Brann [3]. The extension lies in our ability to permit nonlinearities in both the wheel and rail profiles and in the inclusion of spin creep terms. Using this model, we study oscillations through a sophisticated tool, viz., the theory of Hopf bifurcation [15, 18, 21, 25, 28], for it can deal with such nonlinearities with ease. Consequently, we are able to extend, numerically, the analytical results derived earlier [20], by using the algorithm due to Weber [31]. This algorithm is such that we are able to make quantitative decisions concerning the stability of the oscillatory solutions of the linearized problem before the numerical scheme is begun. The quantitative decision concerns the initial value of the solution, and the wheel and rail parameters and results in a considerable saving of the labor expended in computing for we compute stable orbits and not unstable ones.

Lastly, we would like to comment briefly on other methods available to compute periodic solutions of autonomous systems (depending on a parameter). The Galerkin method, due to Urabe [29, 30], seeks a periodic solution to the autonomous system and then finds neighboring orbits by turning the autonomous equations into time-dependent, periodic ones and applying an approximation scheme to calculate these orbits. The describing function method $[19,27]$ replaces the nonlinear terms by equivalent linear terms; the latter are chosen so that the mean square error between the actual output of a nonlinear term and its corresponding linear part is minimized, and it is not always successful [19] in finding the orbits. The averaging method $[2,8]$ consists in finding a co-ordinate transformation so that after averaging, the equations become much less complicated and are therefore easily integrated. We note that recently, the above 
techniques have been employed in $[3,9,10,23]$ and in $[13,16,26]$ to determine the limit cycle of wheelsets and trucks.

We have followed the Hopf bifurcation technique for it concentrates heavily on the effects induced by the parameter, viz., the speed of the wheelset. Failure to be particular about it could result in two or more orbits, rather than a unique one, as the solution to the motion of the system-for an example, see equation (15) below. Secondly, the methods of solving the given problem by using the Hopf bifurcation technique lead directly to the period and phase plane portrait of an orbit as a function of a second parameter $\varepsilon$, with the first parameter appearing in the equations (viz., the speed) depending on this new parameter. Thirdly, apart from the common restrictions of analyticity, Hopf bifurcation is not subject to the requirements of small nonlinearities. As an illustration of this, see the examples 3.1 and 3.2 due to Doedel [12]. In sum, we believe that the Hopf bifurcation theory is versatile, simpler to employ (as will be seen below) and that the algorithm we apply gives a total picture of the system more easily than other approaches available in the literature.

With these remarks, we now turn to a brief description of the way Langford [22] has turned the (local) Hopf bifurcation problem into a two point boundary value problem. For other approaches, see [15, 18, 21, 25, 28]. Although global Hopf bifurcation is not relevant to the axle problem and not discussed here, theorems guaranteeing such phenomena exist and are mentioned in [25], for example.

\section{Hopf bifurcation: Langford's approach}

Suppose one considers the first order system of differential equations:

$$
d \mathbf{x} / d t=\dot{\mathbf{x}}=\mathbf{f}(\mathbf{x}, \Gamma),
$$

depending on a real parameter $\Gamma$. Here, $x$ is an $n$-dimensional (column) vector. Suppose further that

$$
\mathbf{f}(\mathbf{0}, \Gamma)=\mathbf{0}
$$

for all $\Gamma$; that is, the zero solution ( $\equiv$ equilibrium position) is a solution for all values of $\Gamma$.

Hopf bifurcation $[15,18,21,25,28]$ deals with the situation when a periodic solution of period $T$, i.e., $\mathbf{x}(0)=\mathbf{x}(T)$, bifurcates from this zero solution as $\Gamma$ is changed. The period $T$ is, in general, not constant and has to be found from the analysis of the equation (1), subject to the periodicity condition.

Hopf [18] established that a periodic solution bifurcates at $\Gamma=\Gamma_{0}$, if two (sufficient) conditions are satisfied. To describe them, let $\tau=2 \pi t / T$ be the 
non-dimensional time. Then equation (1) can be expressed in terms of $\tau$ and a new parameter $\mu=\Gamma-\Gamma_{0}$ as:

$$
d \mathbf{x} / d \tau=\mathbf{x}^{\prime}=\frac{T}{2 \pi} \hat{\mathbf{f}}(\mathbf{x}, \mu), \quad \hat{\mathbf{f}}(\mathbf{0}, \mu)=\mathbf{0},
$$

where $\mathbf{f}(\mathbf{x}, \Gamma)=\hat{\mathbf{f}}(\mathbf{x}, \mu)$, and the periodicity condition becomes

$$
\mathbf{x}(0)=\mathbf{x}(2 \pi) .
$$

We shall assume that in terms of the new variables $(\mathbf{x}, \mu), T=T(\mu)$ and that

$$
\hat{\mathbf{f}}(\mathbf{x}, \mu)=\mathbf{A}^{0} \mathbf{x}+\mu \mathbf{A}^{1} \mathbf{x}+\mathbf{C}(\mathbf{x})+\mathbf{R}(\mathbf{x}, \mu),
$$

where $\mathbf{A}^{0}, \mathbf{A}^{\mathbf{l}}$ are the matrices:

$$
\begin{aligned}
& A_{i j}^{0}=\left.\frac{\partial \hat{f}_{i}}{\partial x_{j}}\right|_{x_{k}=0 ; \mu=0}, \\
& A_{i j}^{1}=\left.\frac{\partial^{2} \hat{f}_{i}}{\partial \mu \partial x_{j}}\right|_{x_{\curlywedge}=0 ; \mu=0} .
\end{aligned}
$$

The term $\mathbf{C}(\mathbf{x})$ is the cubic term:

$$
6 C_{t}(\mathbf{x})=x_{j} x_{k} x_{m}\left(\frac{\partial^{3} \hat{f}_{i}}{\partial x_{j} \partial x_{k} \partial x_{m}}\right)_{x_{n}=0 ; \mu=0},
$$

and $\mathbf{R}$ is the remainder term, which is small in the following sense:

$$
\begin{aligned}
\left\|\mathbf{R}\left(\mathbf{x}_{1}, \mu_{1}\right)-\mathbf{R}\left(\mathbf{x}_{2}, \mu_{2}\right)\right\| \leqslant & K\left(\left\|\left(\mathbf{x}_{1}, \mu_{1}\right)\right\|^{2}+\left\|\left(\mathbf{x}_{2}, \mu_{2}\right)\right\|^{2}\right) \\
& \times\left\|\left(\mathbf{x}_{1}, \mu_{1}\right)-\left(\mathbf{x}_{2}, \mu_{2}\right)\right\|,
\end{aligned}
$$

where $\|(\mathbf{x}, \mu)\|$ is the Euclidean norm:

$$
\|(\mathbf{x}, \mu)\|=\left[\|\mathbf{x}\|^{2}+\mu^{2}\right]^{1 / 2},
$$

and $K>0$ is a constant. We now return to equation (5) and comment on the expansion. Because $\hat{\mathbf{f}}(\mathbf{0}, \mu)=\mathbf{0}$, no terms in $\mu$ alone can appear. Next, the axle problem has forced us to omit the quadratic terms in $\mathbf{x}$, although they are important in other areas [21,31]. Again, the axle problem has forced us to omit two cubic terms of order $\mu^{2} \mathbf{x}$ and $\mu \mathbf{x x}$. However, these various nonlinearities do not affect the initiation of the bifurcating solution as will be seen next.

For, Hopf's first condition is that:

(i) $\mathrm{A}^{0}$ has a pair of imaginary conjugate eigenvalues $\pm i \beta, \beta>0 . \mathrm{A}^{0}$ has no eigenvalues of the form ni $\beta, n \in\{0,2,3, \ldots\}$, i.e., $i \beta$ is an algebraically simple eigenvalue of $\mathbf{A}^{0}$.

Next, let $\mathbf{A}(\mathbf{0}, \mu)$ denote the matrix:

$$
(\mathbf{A}(\mathbf{0}, \mu))_{\imath \jmath}=\left.\frac{\partial \hat{f}_{i}}{\partial x_{j}}\right|_{x_{k}=0}, \quad \mathbf{A}(\mathbf{0}, 0) \equiv \mathbf{A}^{0},
$$


and let $\delta=\delta(\mu)$ denote the differentiable simple eigenvalue of $\mathbf{A}(0, \mu)$ near $\mu=0$ which coincides with the eigenvalue $i \beta$ of $\mathbf{A}^{0}$ at $\mu=0$. Define

$$
\alpha^{\prime}=\operatorname{Re}\left[\delta^{\prime}(0)\right], \quad \beta^{\prime}=\operatorname{Im}\left[\delta^{\prime}(0)\right] .
$$

That is, near $\mu=0, \delta(\mu)$ is of the form

$$
\delta(\mu)=\alpha^{\prime} \mu+i\left(\beta+\beta^{\prime} \mu\right)+o(\mu) .
$$

Given this equation (13), Hopf's second condition is that:

$$
\alpha^{\prime} \neq 0 \text {. }
$$

This condition is usually expressed as saying that the eigenvalue $\delta(\mu)$ crosses the imaginary axis, at $\mu=0$, with a non-zero (eigenvalue) speed.

To illustrate that the condition (ii) is important, we consider the following $2 \times 2$ system in polar co-ordinates, due to Chefee [7]:

$$
\left.\begin{array}{l}
\dot{r}=r(r-\mu)^{2}(2 \mu-r), \\
\dot{\theta}=1 .
\end{array}\right\}
$$

Rewrite equation (15) in terms of $x=r \cos \theta, y=r \sin \theta$ :

$$
\left[\begin{array}{c}
\dot{x} \\
\dot{y}
\end{array}\right]=\left[\begin{array}{ll}
2 \mu^{3} & -1 \\
1 & 2 \mu^{3}
\end{array}\right]\left[\begin{array}{l}
x \\
y
\end{array}\right]+\text { (nonlinear terms). }
$$

The matrix $\mathbf{A}(\mathbf{0}, \mu)$ of the system (16) is

$$
[\mathbf{A}(\mathbf{0}, \mu)]=\left[\begin{array}{ll}
2 \mu^{3} & -1 \\
1 & 2 \mu^{3}
\end{array}\right]
$$

and its eigenvalue $\delta(\mu)$, which coincides with $i$ at $\mu=0$, is:

$$
\delta(\mu)=2 \mu^{3}+i \text {. }
$$

Thus, the system given by equation (16) violates Hopf's condition (ii), since $\alpha^{\prime}=0$ for the equation (18). A glance at equation (15) shows that if $\mu>0$, there are two distinct periodic orbits given by $r=\mu, r=2 \mu$ respectively. Thus, if one wishes to discuss the behaviour of a system leading to a single periodic orbit for the variable $\mathbf{x}$ when $\mu \neq 0$, Hopf's conditions (i) and (ii) are both absolutely vital. Though Freedman [14] has found a different sufficient condition for a single periodic orbit to bifurcate from the equilibrium position at $\Gamma=\Gamma_{0}$ when Hopf's condition (ii) fails, Freedman's condition is not relevant to our problem for the axle system obeys Hopf's conditions (i) and (ii) as will be seen later.

We now return to the equations (3)-(5). To proceed to further analysis of the system given by equations (3)-(5), consider the linearization of these equations about $(\mathbf{x}, \mu)=(\mathbf{0}, 0)$. Thus we have to solve

$$
\mathbf{x}^{\prime}=\frac{T_{0}}{2 \pi} \mathbf{A}^{0} \mathbf{x}, \quad T_{0}=T(0), \quad \mathbf{x}(0)=\mathbf{x}(2 \pi) .
$$


Let $\mathbf{A}^{0}$ satisfy Hopf's first condition and let $\mathbf{c}=\mathbf{a}+i \mathbf{b}$ be the right complex eigenvector corresponding to the eigenvalue $i \beta$, i.e., $\mathbf{A}^{0} \mathbf{c}=i \beta \mathbf{c}$. Then equation (19) has the solution

$$
\mathbf{x}(\tau)=c e^{\prime \tau}, \quad T_{0}=2 \pi / \beta .
$$

A real basis for the solution space of the equation (19) is given by [22]

$$
\begin{aligned}
& \phi_{1}(\tau)=\mathbf{a} \cos \tau-\mathbf{b} \sin \tau=\operatorname{Re}\left[\mathbf{c} e^{i \tau}\right], \\
& \phi_{2}(\tau)=\mathbf{a} \sin \tau+\mathbf{b} \cos \tau=\operatorname{Im}\left[\mathbf{c} e^{i \tau}\right] .
\end{aligned}
$$

We normalize $\mathbf{c}$ through

$$
\mathbf{a}^{T} \mathbf{a}+\mathbf{b}^{T} \mathbf{b}=2,
$$

where the superscript $T$ denotes the transpose. If $\mathbf{e} \in \mathbf{R}^{n}$ is any non-zero vector with a non-zero projection on the plane spanned by $\{\mathbf{a}, \mathbf{b}\}$, we obtain a uniquely determined (except for sign) solution $\phi(\tau)$ [22] of equation (19) with $\langle\phi, \phi\rangle=1$, and

$$
\mathbf{e}^{T} \phi(0)=0 .
$$

Here, for any two vectors, the inner product is:

$$
\langle\mathbf{u}, \mathbf{v}\rangle=\frac{1}{2 \pi} \int_{0}^{2 \pi} \mathbf{u}(\tau)^{T} \mathbf{v}(\tau) d \tau, \quad\|\mathbf{u}\|^{2}=\langle\mathbf{u}, \mathbf{u}\rangle
$$

Therefore the equations ( 3 ) and (4) can be written as:

$$
\begin{gathered}
\mathbf{x}^{\prime}=\frac{T}{2 \pi} \hat{\mathbf{f}}(\mathbf{x}, \mu), \\
\mathbf{B}_{0} \mathbf{x}(0)+\mathbf{B}_{2 \pi} \mathbf{x}(2 \pi)=\mathbf{0},
\end{gathered}
$$

for all $\mu$, where $\mathbf{B}_{0}$ and $\mathbf{B}_{2 \pi}$ are $(n+1) \times n$ matrices:

$$
\left[\mathbf{B}_{0}\right]=\left[\frac{\mathbf{I}}{\mathbf{e}^{T}}\right], \quad \mathbf{B}_{2 \pi}=\left[\frac{-\mathbf{I}}{\mathbf{0}}\right],
$$

and $I$ is the $n \times n$ unit matrix. Note that in equation (27), the periodicity condition (4) is incorporated. Moreover, the condition $\mathbf{e}^{T} \mathbf{x}(0)=0[18,22]$ also appears in equation (27) in order that one may remove the time-shift in the autonomous system (26).

Now, we note that $\phi(0)$ is a linear combination of $\phi_{1}(0)$ and $\phi_{2}(0)$. Thus, $\boldsymbol{\phi}(0)=a_{1} \mathbf{a}+b_{1} \mathbf{b}$, and $\|\boldsymbol{\phi}(0)\|=1$. Since $\mathbf{e}=a_{2} \mathbf{a}+b_{2} \mathbf{b}+\mathbf{e}^{\perp}$ where $\mathbf{e}^{\perp}$ is orthogonal to $\{\mathbf{a}, \mathbf{b}\}$ and, at least, $a_{2}$ or $b_{2}$ is non-zero, we get from equation (24) that

$$
2 b_{1} b_{2}+\left(a_{1} a_{2}-b_{1} b_{2}\right) \mathbf{a}^{T} \mathbf{a}+\left(a_{1} b_{2}+a_{2} b_{1}\right) \mathbf{a}^{T} \mathbf{b}=0,
$$

where $\mathbf{a}^{T} \mathbf{a}+\mathbf{b}^{T} \mathbf{b}=2$ has been used. Hence, given any initial condition $\phi(0)=$ $a_{1} \mathbf{a}+b_{1} \mathbf{b}$, we can find a vector e to satisfy equation (24). 


\section{Weber's algorithm}

After Langford [22] had constructed an algorithm for finding the periodic solutions to equations (26)-(27), Weber [31] discovered two more. We present a summary of one of his methods below, omitting the proofs.

Firstly, the unique solution to $\mathbf{x}^{\prime}=(T / 2 \pi) \hat{\mathbf{f}}(\mathbf{x}, \mu)$ generates a dynamical system. For such a system, a motion is periodic with a period $2 \pi$ if $\mathbf{x}(0)=\mathbf{x}(2 \pi)$, (see [1]). Therefore, if one can find an initial value $x(0)=\mathbf{s}$ of the above system such that $\mathbf{x}(2 \pi)=\mathbf{s}$, the motion of this system is $2 \pi$-periodic in $\tau$ for all $\tau$. Weber's method consists in finding this initial value as a function of a parameter $\varepsilon$; his algorithm delivers $\mu=\mu(\varepsilon)$ and $T=T(\varepsilon)$ as well so that one can read off $\Gamma(\varepsilon)=\Gamma_{0}+\mu(\varepsilon)$ and the period $T(\varepsilon)$ in $t$ of the original system in equation (1). In addition, in finding these quantities $\mathbf{s}, \mu$ and $T$, one has to solve a set of initial value problems so that the periodic solution of the system itself is found. Thus Weber's method gives a complete picture of all the information that is required.

Now, by $\mathbf{x}=\mathbf{x}(\tau ; \mu, T, \mathbf{s})$, let us denote the unique solution to the initial value problem:

$$
\left.\begin{array}{l}
\mathbf{x}^{\prime}=\frac{T}{2 \pi} \hat{\mathbf{f}}(\mathbf{x}, \mu), \quad 0<\tau<2 \pi \\
\mathbf{x}(0)=\mathbf{s} .
\end{array}\right\}
$$

Define a vector valued mapping $F: \mathbf{R}^{n} \times \mathbf{R}^{2} \rightarrow \mathbf{R}^{n+1}$ through

$$
\mathbf{F}(\mathbf{s}, \mu, T)=\mathbf{B}_{0} \mathbf{s}+\mathbf{B}_{2 \pi} \mathbf{x}(2 \pi ; \mu, T, \mathbf{s}) .
$$

Then, the periodic solution $\mathbf{x}(0)=\mathbf{x}(2 \pi)$ to the equation (30) is equivalent to finding the solution of the finite dimensional, algebraic system

$$
\mathbf{F}(\mathbf{s}, \mu, T)=\mathbf{0} .
$$

To see this, recall that if $\mathbf{x}(2 \pi ; \mu, T, \mathbf{s})=\mathbf{s}$, then $\mathbf{x}(\tau ; \mu, T, \mathbf{s})$ is $2 \pi$-periodic. Clearly, $\mathbf{F}(\mathbf{s}, \mu, T)=\mathbf{0}$ ensures this; the converse is trivial to verify. Now it is easy to see that $\mathbf{F}(\mathbf{0}, \mu, T)=\mathbf{0}$ and therefore we can expand $\mathbf{F}(\mathbf{s}, \mu, T)$ about $\left(\mathbf{0}, 0, T_{0}\right)$ as

$$
\mathbf{F}(\mathbf{s}, \mu, T)=\mathbf{M}_{1} \mathbf{s}+\mu \mathbf{M}_{2} \mathbf{s}+\left(T-T_{0}\right) \mathbf{M}_{3} \mathbf{s}+\text { h.o.t. }
$$

where h.o.t. stands for 'higher order terms' and

$$
\left.\begin{array}{l}
\mathbf{F}_{\mathbf{s}}\left(\mathbf{0}, 0, T_{0}\right)=\mathbf{M}_{1} \\
\mathbf{F}_{\mathrm{s} \mu}\left(\mathbf{0}, 0, T_{0}\right)=\mathbf{M}_{2} \\
\mathbf{F}_{\mathrm{s} r}\left(\mathbf{0}, 0, T_{0}\right)=\mathbf{M}_{3}
\end{array}\right\}
$$


Here the $\mathbf{M}_{i}, i=1,2,3$, are all $(n+1) \times n$ matrices because $\mathbf{F}$ is an $(n+1)$ vector. These matrices can be determined in the following fashion [31]. Let $\boldsymbol{\Phi}(\tau)$ denote the fundamental matrix:

$$
\left.\begin{array}{l}
\boldsymbol{\Phi}^{\prime}=\frac{T_{0}}{2 \pi} \mathbf{A}^{0} \boldsymbol{\Phi}, \\
\boldsymbol{\Phi}(0)=\mathbf{l},
\end{array}\right\}
$$

where I is the $n \times n$ unit matrix. Then, using equations (31), (33)-(35), Weber [31] shows that

$$
\left.\begin{array}{l}
\mathbf{M}_{1}=\mathbf{B}_{0}+\mathbf{B}_{2 \pi} \boldsymbol{\Phi}(2 \pi), \\
\mathbf{M}_{2}=\frac{T_{0}}{2 \pi} \mathbf{B}_{2 \pi} \int_{0}^{2 \pi} \boldsymbol{\Phi}(2 \propto \tau) \mathbf{A}^{\mathbf{l}} \boldsymbol{\Phi}(\tau) d \tau, \\
\mathbf{M}_{3}=\mathbf{B}_{2 \pi} \boldsymbol{\Phi}(2 \pi) \mathbf{A}^{0} .
\end{array}\right\}
$$

Let us now introduce the notation $p=\alpha \phi(0)$, with $\phi(0)$ being the initial value appearing in equation (24) and $\alpha$ chosen so that $\mathbf{p}^{T} \mathbf{p}=1$. Seek solutions to $F(\mathbf{s}, \mu, T)=0$ of equation (33) in the form [31]

$$
\left.\begin{array}{l}
\mathbf{s}(\varepsilon)=\boldsymbol{\varepsilon} \mathbf{p}+\varepsilon^{3} \mathbf{w}(\varepsilon) \\
\mu(\varepsilon)=\varepsilon^{2} \eta(\varepsilon) \\
T(\varepsilon)=T_{0}+\varepsilon^{2} P(\varepsilon)
\end{array}\right\}
$$

along with the orthogonality condition

$$
\mathbf{p}^{T} \mathbf{w}=0
$$

Comparing equations (27) and (35) we see that

$$
\mathbf{M}_{1} \mathbf{p}=\mathbf{0}
$$

because $\phi(2 \pi)=\boldsymbol{\Phi}(2 \pi) \phi(0)=\phi(0)$ and $\mathbf{e}^{T} \mathbf{p}=0$ from equation (24). Using $\mathbf{M}_{1} \mathbf{p}=\mathbf{0}$ in equation (33) leads to $\mathbf{F}\left(\varepsilon \mathbf{p}+\varepsilon^{3}\left((\varepsilon), \varepsilon^{2} \eta \mathbf{w} \varepsilon\right), T_{0}+\varepsilon^{2} P(\varepsilon)\right)=\mathbf{0}$ being written as:

$$
\begin{aligned}
\mathbf{M}_{1} \mathbf{w}(\varepsilon)+ & \eta(\varepsilon) \mathbf{M}_{2} \mathbf{p}+P(\varepsilon) \mathbf{M}_{3} \mathbf{p} \\
& =-\varepsilon^{-3}\left\{(\text { h.o.t. })+\varepsilon^{5} \eta(\varepsilon) \mathbf{M}_{2} \mathbf{w}(\varepsilon)+\varepsilon^{5} P(\varepsilon) \mathbf{M}_{3} \mathbf{w}(\varepsilon)\right\} .
\end{aligned}
$$

Adjoining equation (38) to this, we obtain

$$
\begin{aligned}
{[\mathbf{M}]\left[\begin{array}{c}
\mathbf{w}(\varepsilon) \\
\eta(\varepsilon) \\
P(\varepsilon)
\end{array}\right] } & \equiv\left[\begin{array}{c:c:c}
\mathbf{M}_{1} & \mathbf{M}_{2} \mathbf{p} & \mathbf{M}_{3} \mathbf{p} \\
\hdashline \mathbf{p}^{T} & 0 & 0
\end{array}\right]\left[\begin{array}{c}
\mathbf{w}(\varepsilon) \\
\eta(\varepsilon) \\
P(\varepsilon)
\end{array}\right] \\
& =-\left[\begin{array}{c}
\varepsilon^{-3}\{\cdot\} \\
0
\end{array}\right],
\end{aligned}
$$


where $\varepsilon^{-3}\{\cdot\}$ denotes the right side of equation (40). Since $\mathbf{M}_{1}$ is $(n+1) \times n$, $\mathbf{M}_{2} \mathbf{p}$ and $\mathbf{M}_{3} \mathbf{p}$ are $(n+1) \times 1$ each and $\mathbf{p}^{T}$ is $1 \times n$, the matrix $\mathbf{M}$ is $(n+2) \times$ $(n+2)$. Weber [31] has shown that this matrix $\mathbf{M}$ is non-singular if Hopf's two conditions are satisfied. Using this fact, one can solve for $\{w, \eta, P\}$ through an iteration process for each fixed $\varepsilon$. The iteration starts with

$$
\mathbf{w}^{(0)}(\varepsilon)=0, \quad \eta^{(0)}(\varepsilon)=0, \quad P^{(0)}(\varepsilon)=0 .
$$

Then, on noting that in equation (40), the right side $\{:\}$ is given by

$$
\{\cdot\}=\mathbf{F}-\varepsilon^{3}\left(\mathbf{M}_{1} \mathbf{w}+\eta \mathbf{M}_{2} \mathbf{p}+P \mathbf{M}_{3} \mathbf{p}\right),
$$

we define the iteration process through $(j=0,1,2, \ldots)$ :

$$
\left[\begin{array}{c}
\mathbf{w}^{(\jmath+1)}(\varepsilon) \\
\eta^{(\jmath+1)}(\varepsilon) \\
P^{(\jmath+1)}(\varepsilon)
\end{array}\right]=\left[\mathbf{M}^{-1}\right]\left[\begin{array}{c}
\left\{\mathbf{M}_{1} \mathbf{w}^{(j)}(\varepsilon)+\mathbf{M}_{2} \mathbf{p} \eta^{(\jmath)}(\varepsilon)+\mathbf{M}_{3} \mathbf{p} P^{(\jmath)}(\varepsilon)\right. \\
\left.-\varepsilon^{-3} \mathbf{F}\left(\varepsilon \mathbf{p}+\varepsilon^{3} \mathbf{w}^{(j)}(\varepsilon), \varepsilon^{2} \eta^{(\jmath)}(\varepsilon), T_{0}+\varepsilon^{2} P^{(\jmath)}(\varepsilon)\right)\right\} \\
0
\end{array}\right] .
$$

As is obvious, the above system requires that at each step $\mathbf{F}(\cdot, \cdot, \cdot)$ be evaluated. Since $\mathbf{F}$ is defined through equation (31), we need to solve the initial value problems

$$
\left.\begin{array}{c}
\mathbf{x}^{(\jmath)}=\left(\left(T_{0}+\varepsilon^{2} P^{(\jmath)}(\varepsilon)\right) / 2 \pi\right) \hat{\mathbf{f}}\left(\mathbf{x}^{(j)}, \varepsilon^{2} \eta^{(\jmath)}(\varepsilon)\right), \\
\left.\mathbf{x}^{(\jmath)}\right|_{T=0}=\varepsilon \mathbf{p}+\varepsilon^{3} \mathbf{w}^{(J)}(\varepsilon), \quad j=0,1,2, \ldots,
\end{array}\right\}
$$

to determine $\mathbf{F}$. Therefore, in practice, the iteration works like this from $\varepsilon=0$ :

(i) Choose $\varepsilon$, say $\varepsilon=\varepsilon_{1}$.

(ii) Since $p$ is known, find $x^{(0)}$ from equation (45), by setting $w^{(0)}\left(\varepsilon_{1}\right)=0$, $P^{(0)}\left(\varepsilon_{1}\right)=\eta^{(0)}\left(\varepsilon_{1}\right)=0$.

(iii) Compute $\mathbf{x}^{(0)}\left(2 \pi ; 0, T_{0}\right)$, and hence $\mathbf{F}\left(\varepsilon_{1} \mathbf{p}, 0, T_{0}\right)$.

(iv) Use these in equation (44) to find $w^{(1)}\left(\varepsilon_{1}\right), \eta^{(1)}\left(\varepsilon_{1}\right)$, and $P^{(1)}\left(\varepsilon_{1}\right)$.

(v) Employ these in equation (45) to find $\mathbf{x}^{(1)}$ and go back to step (ii).

After the iterations have converged at $\varepsilon=\varepsilon_{1}$, choose a new value $\varepsilon=\varepsilon_{2}$ and go back to step (ii).

In this way, by solving a set of $n$ initial value problems at each step of the iteration and solving the systems of equations (44) at each iteration step, we generate the solution set $\{\mathbf{x}(\varepsilon), \mathbf{s}(\varepsilon), \mu(\varepsilon), T(\varepsilon)\}$. Convergence of the scheme occurs over a reasonable range of $\varepsilon$, which depends on the original equation (3). We do not list this in detail here-see $[22,31]$ for further information.

Lastly, since we begin with $\left\{w^{(0)}(\varepsilon), \eta^{(0)}(\varepsilon), P^{(0)}(\varepsilon)\right\}=\{0,0,0\}$, the first iteration $\left\{\mathbf{w}^{(1)}(\varepsilon), \eta^{(1)}(\varepsilon), P^{(1)}(\varepsilon)\right\} \neq\{0,0,0\}$ provided $\varepsilon^{-3} \mathbf{F}\left(\varepsilon p, 0, T_{0}\right) \neq \mathbf{0}$ in equation (44). Now because of equation (39), $F\left(\varepsilon p, 0, T_{0}\right)$ is not linear in $\varepsilon$ and so we need $\mathbf{F}\left(\varepsilon \mathbf{p}, 0, T_{0}\right)$ to be non-zero in $\varepsilon^{3}$. This can be shown to be equivalent to demanding that $\mathbf{C}(\mathbf{x})$ in equation (8) be non-zero. Of course, if $\mathbf{C}(\mathbf{x})$ is zero then we will 
have to seek solutions of the form $\left\{\varepsilon \mathbf{p}+\varepsilon^{k} w(\varepsilon), \varepsilon^{k-1} \eta(\varepsilon), T_{0}+\varepsilon^{k-1} P(\varepsilon)\right\}$ rather than the assumed set in equation (37), with $k \geqslant 4$ depending on the first non-zero, nonlinear term in the expansion of $\hat{\mathbf{f}}(\mathbf{x}, \mu)$ in equation (5). On the other hand if $\hat{\mathbf{f}}(\mathbf{x}, \mu)$ contains quadratic terms then the solution to be sought is of the form $\left\{\varepsilon p+\varepsilon^{2} \mathbf{w}(\varepsilon), \varepsilon \eta(\varepsilon), T_{0}+\varepsilon P(\varepsilon)\right\}$ as is obvious.

We now turn to the axle system and cast it in a form amenable to the theory developed so far.

\section{The axle system}

We model the axle following Brann [3], but with some modifications for we take into account the axle load $W$, and spin creep coefficients following Burton and Whitman [5,6] and Hobbs and Pearce [17]. The equations of motion are (see the Nomenclature section at the end of the paper for definitions of the symbols):

$$
\begin{gathered}
m \ddot{y}+f k(\dot{y} / V-\theta)+2 f_{23} \dot{\theta} / V+(W / 2)\left(\tan \delta_{2}-\tan \delta_{1}\right)=0, \\
I \ddot{\theta}+\left(2 f b^{2} / V\right) \dot{\theta}-2 f_{23}(\dot{y} / V-\theta)=(f b \Omega / V)\left(r_{1}-r_{2}\right) .
\end{gathered}
$$

The lateral and longitudinal creep coefficients are assumed equal and are given by $f$, and $f_{23}$ is the spin/lateral creep coefficient. We can safely neglect the spin creep coefficient $f_{33}$ which is much smaller than $f b^{2}$. The differences compared with Brann's model [3] are the inclusions of the lateral/spin creep terms, the total weight $W$, and we also allow for non-conical wheel profiles. We shall assume that

$$
\left.\begin{array}{l}
\tan \delta_{2}-\tan \delta_{1}=e_{1} y+e_{3} y^{3} \\
r_{1}-r_{2}=q_{1} y+q_{3} y^{3}
\end{array}\right\}
$$

Using the variables

$$
\left.\begin{array}{c}
z=y / b, \quad A=f k / m b, \quad \Gamma=f k / m V, \\
\Sigma=2 m b^{2} / I k, \quad \rho=f_{23} / f b, \\
h_{s}=(W / 2 m) e_{s} b^{s+1}, \quad p_{s}=-(f / I r) q_{s} b^{s+1}, \quad s=1,3, \\
h(z)=h_{1} z+h_{3} z^{3}, \quad p(z)=-p_{1} z-p_{3} z^{3},
\end{array}\right\}
$$

we turn the system of equations (46) into

$$
\left.\begin{array}{l}
\ddot{z}+\Gamma \dot{z}-A \theta+\rho \Gamma \dot{\theta}+h(z)=0, \\
\ddot{\theta}+\Gamma \Sigma \dot{\theta}+A \Sigma \rho \theta-\rho \Sigma \Gamma \dot{z}=p(z) .
\end{array}\right\}
$$


Let $z=x_{1}, \dot{z}=x_{2}, \theta=x_{3}, \dot{\theta}=x_{4}, \tau=2 \pi t / T$. Then equation (49) becomes:

$$
\left[\mathbf{x}^{\prime}\right]=\frac{T}{2 \pi}\left[\begin{array}{l}
x_{2} \\
-h\left(x_{1}\right)-\left(\Gamma_{0}+\mu\right) x_{2}+A x_{3}-\rho\left(\Gamma_{0}+\mu\right) x_{4} \\
x_{4} \\
p\left(x_{1}\right)+\Sigma \rho\left(\Gamma_{0}+\mu\right) x_{2}-A \Sigma \rho x_{3}-\left(\Gamma_{0}+\mu\right) \Sigma x_{4}
\end{array}\right],
$$

where we have put $\Gamma=\Gamma_{0}+\mu$. Hence, equation (50) is of the form given by equation (3). The matrices $\mathbf{A}(\mathbf{0}, \mu)$ and $\mathbf{A}^{\prime}$ of equation (50) are:

$$
\begin{gathered}
{[\mathbf{A}(\mathbf{0}, \mu)]=\left[\begin{array}{cccc}
0 & 1 & 0 & 0 \\
-h_{1} & -\left(\Gamma_{0}+\mu\right) & A & -\rho\left(\Gamma_{0}+\mu\right) \\
0 & 0 & 0 & 1 \\
-p_{1} & \rho \Sigma\left(\Gamma_{0}+\mu\right) & -\rho \Sigma A & -\left(\Gamma_{0}+\mu\right) \Sigma
\end{array}\right],} \\
{\left[\mathbf{A}^{\prime}\right]=\left[\begin{array}{cccc}
0 & 0 & 0 & 0 \\
0 & -1 & 0 & -\rho \\
0 & 0 & 0 & 0 \\
0 & \rho \Sigma & 0 & -\Sigma
\end{array}\right]}
\end{gathered}
$$

Now, in equation (50), if we put $\mathbf{x}^{\prime}=\mathbf{0}$, we get that $x_{2}=0, x_{4}=0$ on the righic side. Moreover, $p\left(x_{1}\right)-A \Sigma \rho x_{3}=0$ and $A x_{3}-h\left(x_{1}\right)=0$ as well. These equations have $x_{1}=0$ as the only (real) solution if and only if $\rho \Sigma h_{1}+p_{1}$ and $\rho \Sigma h_{3}+p_{3}$ are of the same sign. Hence, we assume that $p_{1}+\rho \Sigma h_{1}>0, p_{3}+$ $\rho \Sigma h_{3}>0$ in what follows - note that Brann [3] has taken $p_{1}>0$, with $p_{3}=0$ and $p=0$. With $x_{1}=0, h\left(x_{1}\right)=0$ trivially and hence $x_{3}=0$. Thus, if

$$
\left.\begin{array}{l}
p_{1}+\rho \Sigma h_{1}>0, \\
p_{3}+\rho \Sigma h_{3}>0,
\end{array}\right\}
$$

the system (50) has the unique solution $\mathbf{x}=\mathbf{0}$, if $\mathbf{x}^{\prime}=\mathbf{0}$, for all $\Gamma\left(=\Gamma_{0}+\mu\right)$. Because $\mathbf{x}=\mathbf{0}$ is the unique solution, there are no non-trivial equilibrium positions for the axle. Of course the converse, namely $\mathbf{x}=\mathbf{0}$, is always a solution of the system (50).

Now, we return to the matrix $\mathbf{A}(0, \mu)$ in equation (51) and note that the matrix $\mathbf{A}^{0} \equiv \mathbf{A}(\mathbf{0}, 0)$ has the eigenvalues:

$$
\pm i \beta, \quad \lambda_{1} \pm i \lambda_{2},
$$

where

$$
\begin{aligned}
& \beta=\left(\frac{h_{1} \Sigma-\rho p_{1}}{\Sigma+1}\right)^{1 / 2}, \\
& \lambda_{1}=-\Gamma_{0}(\Sigma+1) / 2,
\end{aligned}
$$


provided

$$
\Gamma_{0}^{2}=\frac{A\left(p_{1}+\rho \Sigma h_{1}\right)}{\beta^{2} \Sigma\left(\rho^{2}+1\right)}-\frac{A \rho \Sigma(\Sigma+1)+h_{1}+p_{1} \rho}{\Sigma(\Sigma+1)\left(\rho^{2}+1\right)}>0 .
$$

We assume that $\Gamma_{0}$ obeys equation (57) from now on. The fact that $\Gamma_{0}$ and $\beta$ must be real imposes inequalities on the parameters $p_{1}, h_{1}$, but these inequalities are satisfied for the standard values assumed below. We choose $\beta>0$ and $\Gamma_{0}>0$.

The eigenvalue $\sigma(\mu)$ of $\mathbf{A}(\mathbf{0}, \mu)$ which coincides with $i \beta$ at $\mu=0$ is given by:

$$
\delta(\mu)=\alpha^{\prime} \mu+i\left(\beta+\beta^{\prime} \mu\right),
$$

where $\alpha^{\prime}$ is given by

$$
\begin{aligned}
\alpha^{\prime}=-\beta^{2} \Gamma_{0}^{2} \Sigma(\Sigma+1)\left(\rho^{2}+1\right) /\left\{\beta^{2} \Gamma_{0}^{2}(\Sigma+1)^{2}\right. \\
\left.+\left[-h_{1}+\frac{h_{1}+\rho p_{1}}{\Sigma+1}+\frac{A\left(p_{1}+\Sigma \rho h_{1}\right)(\Sigma+1)}{h_{1} \Sigma-\rho p_{1}}\right]^{2}\right\} .
\end{aligned}
$$

Thus $\alpha^{\prime} \neq 0$ and the system given by equation (50) obeys Hopf's conditions (i) and (ii).

Taking $\left[\mathbf{e}^{r}\right]=[1,0,0,0]$, one can show from equations (20)-(23) that the unique solution $\phi(\tau)$ to the linearized problem $\mathbf{x}^{\prime}=\left(T_{0} / 2 \pi\right) \mathbf{A}^{0} \mathbf{x}, \mathbf{x}(0)=\mathbf{x}(2 \pi)$, of the system (50), is:

$$
\phi(\tau)=\mathbf{a} \sin \tau+\mathbf{b} \cos \tau
$$

where

$$
[\mathbf{c}]=[\mathbf{a}]+i[\mathbf{b}]=c_{1}\left[\begin{array}{l}
1 \\
i \beta \\
\frac{h_{1}-\beta+i \beta \Gamma_{0}}{A-i \beta \rho \Gamma_{0}} \\
\frac{-\beta^{2} \Gamma_{0}+i \beta\left(h_{1}-\beta^{2}\right)}{A-i \beta \rho \Gamma_{0}}
\end{array}\right],
$$

and $c_{1}$ is determined from $\mathbf{a}^{T} \mathbf{a}+\mathbf{b}^{T} \mathbf{b}=2$, or

$$
\begin{aligned}
c_{1}^{2}\left(\beta^{2}+1\right)\{[ & \left.A\left(h_{1}-\beta^{2}\right)-\rho \beta^{2} \Gamma_{0}^{2}\right]^{2}+ \\
& {\left[A^{2}+\beta^{2} \rho^{2} \Gamma_{0}^{2}\right]^{2} } \\
& \left.+\beta^{2} \Gamma_{0}^{2}\left[A+\rho\left(h_{1}-\beta^{2}\right)\right]^{2}\right\} \\
=2\left[A^{2}+\beta^{2} \rho^{2} \Gamma_{0}^{2}\right]^{2} . &
\end{aligned}
$$

Note that $\mathbf{e}^{T} \mathbf{a}=c_{1} \neq 0$ and, moreover,

$$
\mathbf{p} \equiv \alpha \phi(0)=\alpha \mathbf{b}, \quad \mathbf{p}^{T} \mathbf{p}=1 .
$$


We now turn to the question of the orbital stability of $\phi(\tau)$.

If $\mathbf{c}^{L}$ is the left eigenvector of $\mathrm{A}^{0}$ corresponding to $i \beta$, i.e. $i \beta \mathbf{c}^{L}=\mathbf{c}^{L} \mathbf{A}^{0}$, and if we normalize $c^{L}$ with respect to $\mathrm{c}$ so that $c_{t}^{L} c_{t}=1$, then it can be shown as in [20] that the stability condition of Poore [28] reduces to a simple algebraic expression. Indeed, if

$$
\operatorname{Re}\left\{\left(h_{3} c_{2}^{L}+p_{3} c_{4}^{L}\right) c_{1}^{2} \bar{c}_{1}\right\}>0,
$$

where $\operatorname{Re}$ denotes the real part, then $\phi(\tau)$ is orbitally stable. Here, $\bar{c}_{1}$ is the conjugate of $c_{1}$, but the latter being real, $\bar{c}_{1}=c_{1}$. So, we are left with a relation connecting $h_{3}, p_{3}, c_{2}^{L}$ and $c_{4}^{L}$. A calculation using $\mathbf{A}^{0}=\mathbf{A}(0,0)$ from equation (51) shows that

$$
c_{2}^{L}=c_{4}^{L} \times \frac{A \rho \Sigma-\beta^{2}+i \beta \Gamma_{0} \Sigma}{A-i \beta \rho \Gamma_{0}}
$$

and therefore $c_{2}^{L}$ is known if $c_{4}^{L}$ is, since the other constants in equation (65) are. Next, using $c_{t}^{L} c_{t}=1$, we determine $c_{4}^{L}$ through equations (61), (62) and (65) as follows:

$$
\begin{aligned}
& \left(A-i \beta \rho \Gamma_{0}\right) / c_{4}^{L}=\left(\Gamma_{0}+2 i \beta\right)\left(A \rho \Sigma-\beta^{2}+i \beta \Gamma_{0} \Sigma\right) \\
& \quad-\rho \Sigma \Gamma_{0}\left(A-i \beta \rho \Gamma_{0}\right)-\beta^{2} \Gamma_{0}+i \beta\left(h_{1}-\beta^{2}\right) \\
& \quad+\left(\Gamma_{0} \Sigma+i \beta\right)\left(h_{1}-\beta^{2}+i \beta \Gamma_{0}\right) \\
& \quad+\rho \Gamma_{0}\left(A \rho \Sigma-\beta^{2}+i \beta \Gamma_{0} \Sigma\right)\left(h_{1}-\beta^{2}+i \beta \Gamma_{0}\right) /\left(A-i \beta \rho \Gamma_{0}\right) .
\end{aligned}
$$

We can verify now from the inequality (64) whether the values of $h_{3}$ and $p_{3}$ used there lead to periodically stable solutions. Only those values of $h_{3}$ and $p_{3}$ leading to orbital stability of $\phi(\tau)$ were used in our numerical scheme.

To answer the stability of larger amplitude oscillations, a theorem employing Floquet theory is available [15, page 42]. In this paper, we have not explored the stability of the large amplitude orbits, although this is being done for a more complicated model under study.

Before moving on to the numerical values employed in the present work, it is worth noting that the non-dimensional time used in Hopf bifurcation is not defined by $\tau=V t / b$ as is commonly assumed in the literature on axle problems [3]. This is because there is no a priori reason to believe $T$ is inversely proportional to $V$, which would be the case if one uses $\tau=V t / b$. In contrast, by defining $\tau=2 \pi t / T$ and finding $V$ and $T$ as a function of $\varepsilon$, we permit a more general relation to exist between $V$ and $T$. 


\section{Numerical values}

For the axle system given by equation (50), the following constants were used or calculated from Brann [3].

$$
\begin{gathered}
b=71.12 \mathrm{~cm}, \quad r=45.72 \mathrm{~cm}, \quad g=981.456 \mathrm{~cm} / \mathrm{sec}^{2}, \\
k=2, \quad I=m b^{2}, \quad \Sigma=1 .
\end{gathered}
$$

We also assume a total axle load of

$$
W=30,000 \mathrm{lb}=133,500 \mathrm{~N},
$$

and a wheelset mass

$$
m=907 \mathrm{~kg} \text {. }
$$

The creep coefficients are discussed by Burton and Whitman $[5,6]$ and are given by

$$
f=\mu W / 2, \quad f_{23} \equiv f_{L \theta}=\mu_{L \theta} W b / 2,
$$

where $\mu, \mu_{L \theta}$ are the normalized creep coefficients with values

$$
\mu=200, \quad \mu_{L}=0.45 \text {. }
$$

We have taken $\mu_{L \theta}$ from [6], with $\mu$ from the Figure 3 of [4]. Now,

$$
\left.\begin{array}{l}
f=3 \times 10^{6} \mathrm{lb}=13.35 \times 10^{6} \mathrm{~N}, \\
f_{23}=15750 \mathrm{lb} . \mathrm{ft}=21364.5 \mathrm{Nm},
\end{array}\right\}
$$

and

$$
\rho=\mu_{L \theta} / \mu=0.00225 \text {. }
$$

Brann [3] chooses for $h_{1}, p_{1}$ the values $h_{1}=0.69$ and $p_{1}=1.073$. However we choose values which are larger by the factors $W / m g$ and $\mu W / m g$ respectively to obtain

$$
h_{1}=10.35, \quad p_{1}=3219
$$

and similarly $A=13.8$, as chosen by Brann, is increased by the factor $\mu \mathrm{W} / \mathrm{mg}$, so we have

$$
A=41400 \text {. }
$$

We calculate then

$$
\beta=1.246, \quad \Gamma_{0}=9261.6,
$$

and the critical speed is $V_{0}=3.17 \mathrm{~m} / \mathrm{sec}$.

The parameters $A, h_{1}, p_{1}, \beta^{2}, \Gamma_{0}^{2}$ have the dimension of $\sec ^{-2} ; k$ and $\Sigma$ are dimensionless. Equation (62) yields for $c_{1}$ the value

$$
c_{1}=0.852 \text {. }
$$


From equations (65)-(66) we obtain

$$
\left(A-i \beta \rho \Gamma_{0}\right) / c_{4}^{L}=-5.76 \times 10^{4}+i 2.14 \times 10^{8},
$$

which is used in the inequality (64), and we are led to choose $h_{3}$ and $p_{3}$ such that

$$
p_{3}<311.01 h_{3} \text {. }
$$

In Brann's work [3] it was assumed that $p_{3}=0$, but we take $p_{3}>0$ for $p_{1}>0$ and $h_{3}$ is fixed by anpealing to the above inequality. In the numerical work wo have chosen

$$
\left.\begin{array}{l}
h_{3} / h_{1}=0.5,1,5 \\
p_{3} / p_{1}=0,0.2
\end{array}\right\}
$$

These values obey the restriction (76).

Employing the numerical values above and calculating the matrices $\mathbf{M}_{1}, \mathbf{M}_{2}$ and $\mathbf{M}_{3}$ for the axle system (50), we found the periodic motions for the yaw and lateral displacements by using Weber's algorithm [31] as described earlier.

\section{Numerical results and discussion}

As pointed out in [3], if the wheels are conical and the rails flat, then in equation (48) $h(z)$ and $p(z)$ are both linear in $z$. To allow for non-conical wheels and non-flat rails, it has been assumed here that both $h(z)$ and $p(z)$ are cubic in z. Now, because $r_{1}-r_{2}$ is expressed in terms of $p(z)$, the parameters $p_{1}$ and $p_{3}$

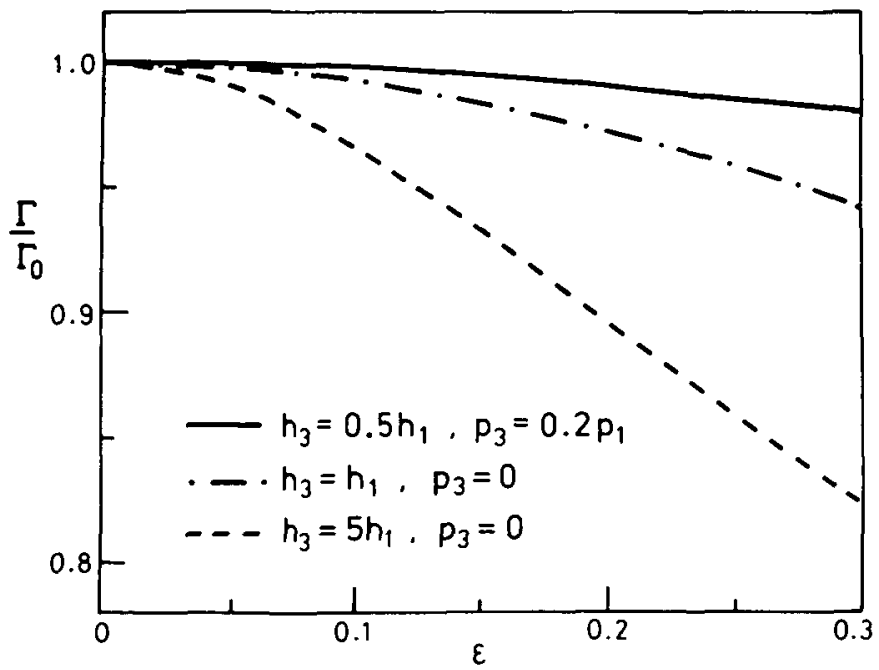

Figure 1. The reciprocal of the speed $\Gamma / \Gamma_{0}\left(\equiv V_{0} / V\right)$ as a function of the parameter $\varepsilon$. 


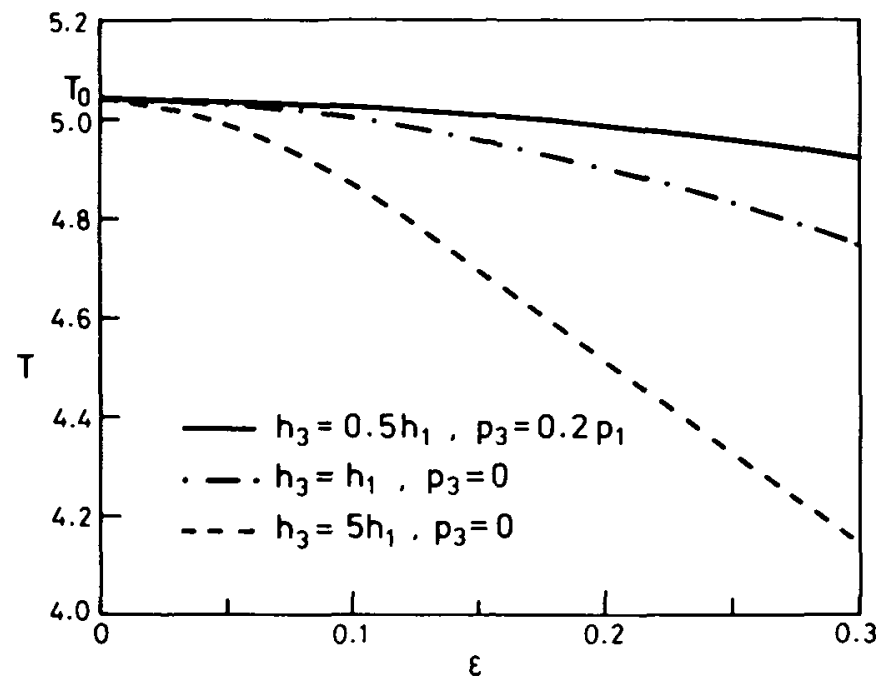

Figure 2. The period of oscillation $T$ as a function of the parameter $\varepsilon$.

have been identified with the wheel profile while the other two, viz., $h_{1}$ and $h_{3}$, with the rails. However, it must be stressed that the geometry of the wheel-rail contact is much too complex for the above simplistic identification of the parameters with the wheel-rail profiles to be anything more than a suggestive one.

Turning now to the discussion of the figures, the first two give a picture of the way the forward speed and the period of oscillation change with the computation parameter $\varepsilon$, for specified values of $h_{3} / h_{1}$ and $p_{3} / p_{1}$. To be specific, Figure 1 is the plot of $\Gamma / \Gamma_{0}$ as a function of $\varepsilon$, for the ratios $h_{3} / h_{1}=0.5,1,5$ and $p_{3} / p_{1}=0$ and 0.2. We have $\Gamma / \Gamma_{0}=V_{0} / V$ from equation (48), where $V_{0}$ is the critical speed $2 \mathrm{f} / \mathrm{m} \Gamma_{0} \sim 3.17 \mathrm{~m} / \mathrm{s}$, and so Figure 1 shows a plot of the reciprocal of the speed as a function of $\varepsilon$. We see in general that $V$ increases with $\varepsilon$. In Figure 2, we have a plot of the period $T$ as a function of $\varepsilon$ over a range of wheel and rail parameters. The period decreases as $V$ (or $\varepsilon$ ) increases and is strongly affected by changes in $h_{3}, i . e$. in the rail profile. In Figures 1 and 2 the plots for $h_{3} / h_{1}=1, p_{3}=0$ differ only slightly from those for $h_{3} / h_{1}=1, p_{3} / p_{1} \doteq 0.2$ (not shown). It may, therefore, be claimed that for a given forward speed, the rail profile has a strong influence on the period of oscillation, pushing the period down as the rail deviates from a flat head.

Figures 3 and 4 yield the phase plane plots for the lateral motion $(z, \dot{z})$ for $h_{3} / h_{1}=1,5$ and $p_{3}=0$. The symmetrical shape of the figures is preserved as $h_{3}$ varies, but the oscillations become more pronounced for smaller $h_{3}$. A similar effect occurs for the yaw motion $(\theta, \dot{\theta})$ plots, which are also symmetric (see 


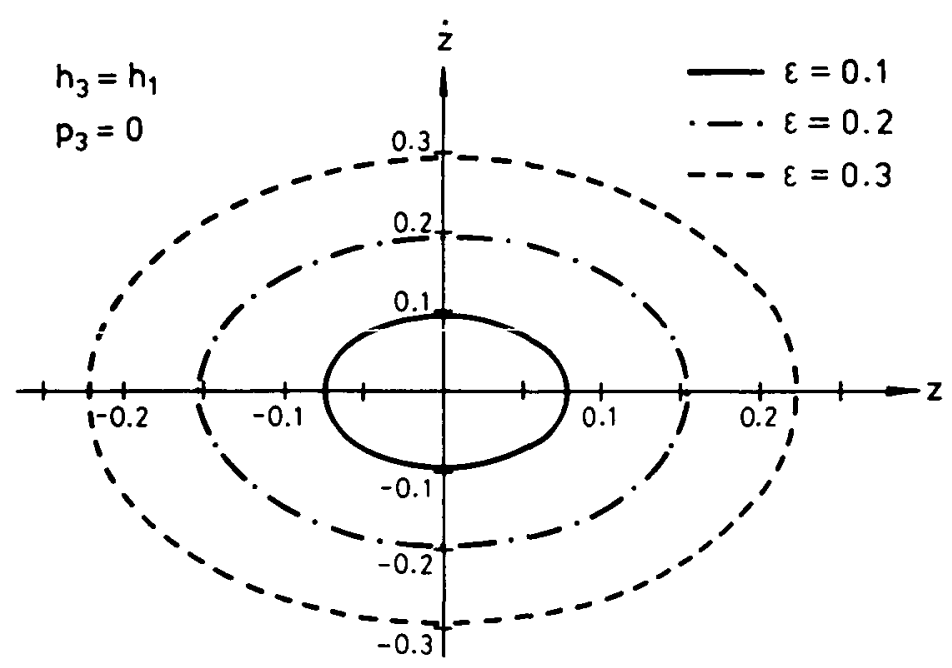

Figure 3. Phase plane plot of the lateral motion, depending on the parameter $\varepsilon$, for a conical wheel with $h_{3}=h_{1}$.

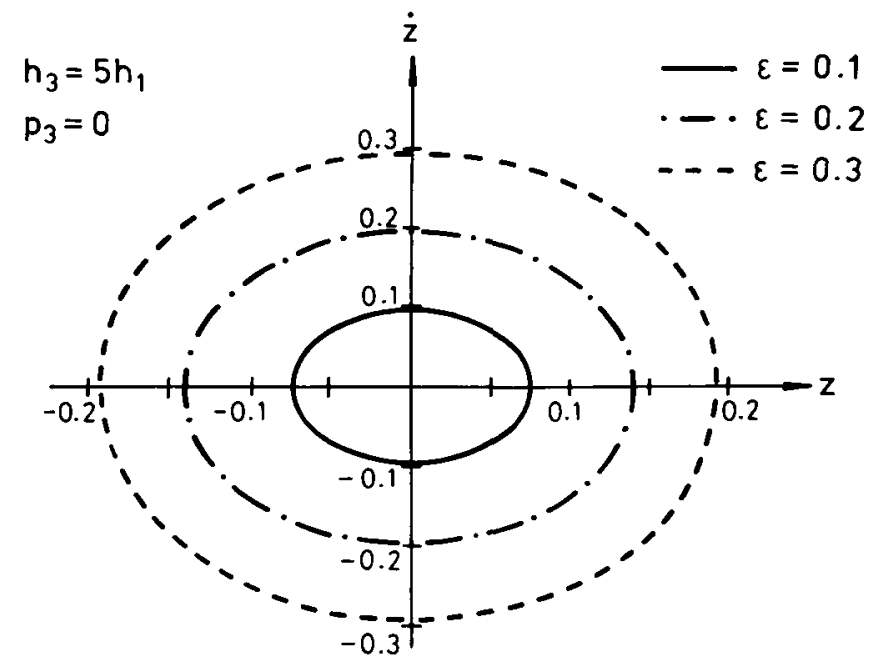

Figure 4. Phase plane plot of the lateral motion, depending on the parameter $\varepsilon$, for a conical wheel with $h_{3}=5 h_{1}$. 


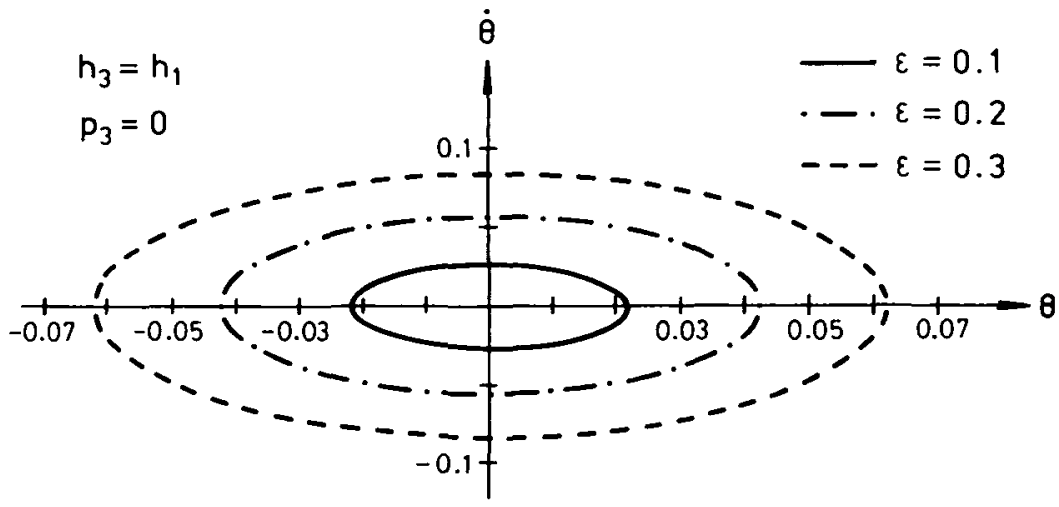

Figure 5. Phase plane plot of the yaw motion, depending on the parameter $\varepsilon$, for a conical wheel $h_{3}=h_{1}$.

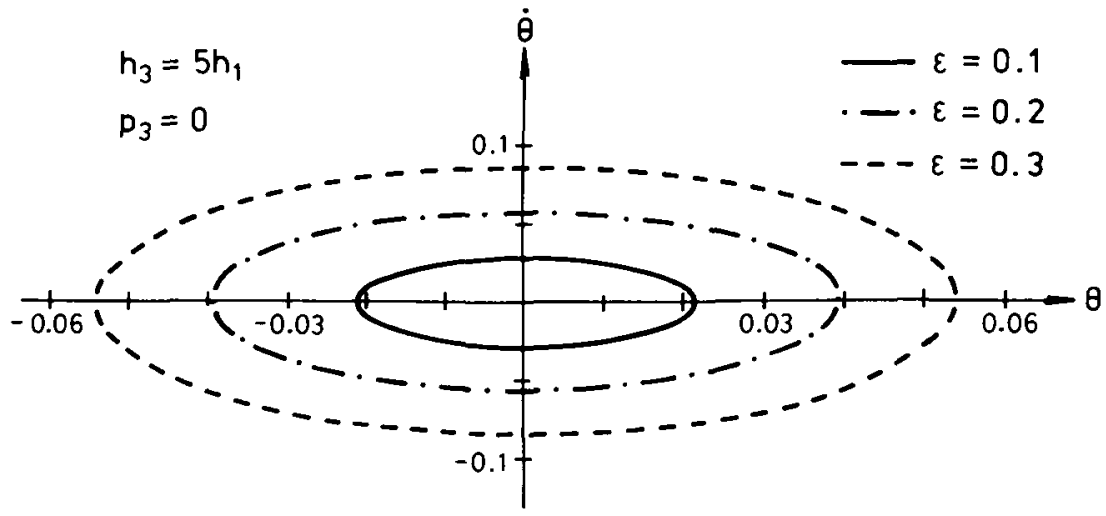

Figure 6. Phase plane plot of the yaw motion, depending on the parameter $\varepsilon$, for a conical wheel with $h_{3}=5 h_{1}$.

Figures 5 and 6). There is very little variation for different values of $p_{3}$; the plots for $h_{3} / h_{1}=1, p_{3} / p_{1}=0.2$ (not shown) are almost identical with those for $h_{3} / h_{1}=1, p_{3}=0$. In conclusion, both $(z, \dot{z})$ and $(\theta, \dot{\theta})$ plots show that the lateral and yaw motion amplitudes increase as the forward speed increases, for any given rail and wheel profile. While the effect of wheel curvature on the lateral motions seems to be non-existent, its effect on the yaw motion amplitude and the 
period is to increase them very slightly as the wheel profile changes from a conical to a curved profile. On the other hand, the effect of rail curvature on the lateral amplitude, for instance, is significant; the larger the curvature the smaller the amplitude for a given forward speed.

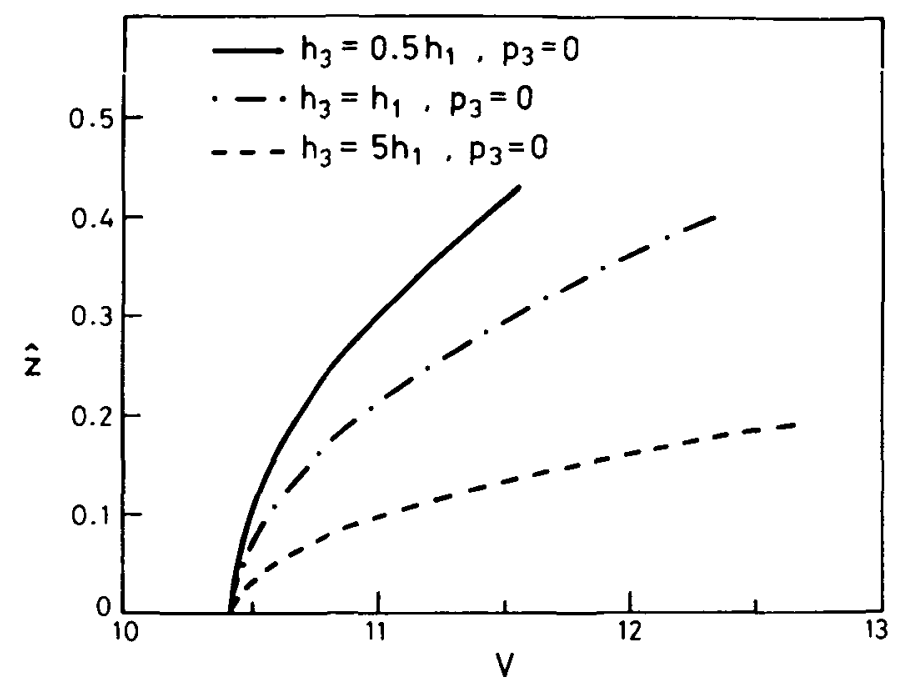

Figure 7. Amplitude of the lateral motion $\hat{z}$ as a function of forward speed (expressed in $\mathrm{ft} / \mathrm{sec}$ ), depending on the rail profile for a conical wheel.

In Figure 7, we have plotted the results for the amplitude $z$ as a function of $V$ for the values $h_{3} / h_{1}=0.5,1$ and 5 , for the case of a conical wheel $\left(p_{3}=0\right)$. Evidently the amplitude decreases as the nonlinearity of the rail profile increases i.e. the motion is more stable for nonlinear rail profiles. However, in each case, since there are no restrictions due to a flange force the amplitude becomes large even for small increases in speed. This situation arises in Brann's case [3] as well - see Figure 6 of that paper.

\section{Nomenclature}

$b$

$f$

$f_{23}$

$f_{33}$ semi-gauge of track

lateral/longitudinal creep coefficient

spin/lateral creep coefficient

spin creep coefficient 


$h_{1}, h_{3}$
$m$
$p_{1}, p_{3}$
$r$
$r_{1}, r_{2}$
$y$
$z$
$\dot{z}$
$\hat{z}$
$I$
$V$
$W$
$\delta_{1}, \delta_{2}$

$\dot{\theta}$
$\Omega$

$h_{1}, h_{3}$

$p_{1}, p_{3}$

$r_{1}, r_{2}$ rail profile parameters

mass of wheelset

wheel profile parameters

radius of contact of both wheels of undis-

placed axle

radii of contact of left and right (rear view)

wheels respectively of displaced wheelset

lateral displacement

non-dimensional lateral displacement

rate of change of $z$

amplitude of $z$

moment of inertia of wheelset about the vertical axis through the center of mass

forward speed along the track

axle load

inclinations of the tangents at points of contact of left and right wheels (rear view) respectively to the horizontal plane yaw angle yaw angular velocity angular velocity of wheelset $=V / r$

\section{Acknowledgements}

We wish to thank Professor W. F. Langford for acquainting one of us (R. R. Huilgol) with Professor H. Weber and Professor Weber for sending us a copy of his paper prior to publication. Further, the work of R. Janus was supported by a Flinders University Research Fellowship and that of $M$. Lohe by an Australian Research Grants Scheme grant awarded to one of us (R. R. Huilgol). We thank these bodies for their assistance and the reviewers for suggesting some improvements.

\section{References}

[1] N. P. Bhatia and G. P. Szegö, Stability theory of dynamıcal systems (Springer-Verlag, New York, 1970).

[2] N. N. Bogoliubov and Y. A. Mitropolsky, Asymptotic methods in the theory of non-linear oscillations (Hindustan Publishing, Delhi, 1961). 
[3] R. P. Brann, "Some aspects of the hunting of a rallway axle", J. Sound and Vibration 4 (1966), 18-32.

[4] T. D. Burton, "Nonlinear effects of contact geometry on railway vehicle wheelset motions", Ph.D. Thesis, Department of Mechanical Engineering and Applied Mechanics, University of Pennsylvania, 1976.

[5] T. D. Burton and A. M. Whitman, "Nonlinear kinematics of wheel-rail contact", J. Appl. Mech. 45 (1978), 664-668.

[6] T. D. Burton and A. M. Whitman, "Nonlinear contact geometry effects on wheelset dynamics", J. Appl. Mech. 47 (1980), 155-160.

[7] N. Chafee, "The bifurcation of one or more closed orbits from an equilibrium point of an autonomous differential system", J. Differential Equations 4 (1968), 661-679.

[8] S -N. Chow and J. Mallet-Paret, "Integral averaging and bifurcation", J. Differential Equations 26 (1977), 112-159.

[9] N. K. Cooperrider, "The huntıng behavior of conventional railway trucks", Trans. ASME Ser. B. J. Engrg. for Industry 94 (1972), 752-762.

[10] A. D. DePater, "The approximate determination of the hunting movement of a railway vehicle by the aid of the method of Krylov and Bogoljubov", Appl. Sct. Res. A 10 (1961), 205-228.

[11] A. D. DePater, "A non-linear model of a single wheelset moving with constant speed on a purely straight track", Internat. J. Non-Linear Mech. 15 (1980), 315-324.

[12] E. Doedel, "AUTO: A program for the automatıc bifurcation analysis of autonomous systems", Congressus Numerantium 30 (1981), 265-284.

[13] A. F. D'Souza and P. Caravavatna, "Analysis of nonlinear hunting vibrations of rail vehicle trucks”, Trans. ASME Ser. L. J. Mech. Design 102 (1980), 77-85.

[14] H. I. Freedman, "On a bifurcation theorem of Hopf and Friedrichs", Canad. Math. Bull. 20 (1977), 95-102.

[15] B. D. Hassard, N. D. Kazarinoff and Y.-H. Wan, Theory and applications of Hopf bifurcation (Cambridge University Press, Cambridge, 1981).

[16] W. Hauschild, "The application of quasilinearization to the limit cycle behaviour of the nonlinear wheel-rail systems", Vehicle System Dynam. 8 (1979), 122-128.

[17] A. E. W. Hobbs and T. G Pearce, "The lateral dynamics of the linear induction motor test vehicle", Trans. ASME Ser G J. Dynamic Systems Measurement Control 96 (1974), 145-157

[18] E. Hopf, "Abzweigung einer penodischer Lösung von einer stationären Lösung eines Differentialsystems", Ber. Math. Phys. Kl. Sächs Akad. Wiss. Leipzıg 94 (1942), 3-22. (For a translation, see [25].)

[19] J. C. Hsu and A. U. Meyer, Modern control principles and applications (McGraw-Hill, New York, 1968).

[20] R R. Hullgol, "Hopf-Friedrichs bifurcation and the hunting of a railway axle", Quart. Appl. Math. 36 (1978), 85-94.

[21] G. Iooss and D D. Joseph, Elementary stability and bifurcation theory (Springer-Verlag, New York, 1980).

[22] W. F. Langford, "Numencal solution of bifurcation problems for ordinary differential equations", Numer Math. 28 (1977), 171-190.

[23] E H. Law and R. S. Brand, "Analysis of the nonlinear dynamics of a railway vehıcle wheelset", Trans. ASME Ser. G J. Dynamic Systems Measurement Control 95 (1973), 28-35.

[24] E. H Law and N. K. Cooperrider, "A survey of railway vehicle dynamics research", Trans. ASME Ser. GJ. Dynamic Systems Measurement and Control 96 (1974), 132-146.

[25] J. E. Marsden and M. McCracken, The Hopf bifurcation and its applications (Springer-Verlag. New York, 1976).

[26] D. Moelle, H. Steinborn and R. Gasch, "Computation of limit cycles of a wheelset using a Galerkin method", Vehicle System Dynam. 8 (1979), 168-171.

[27] K. Ogata, Modern control engineering (Prentice-Hall, Englewood Cliffs, N.J., 1970). 
[28] A. B. Poore, "On the theory and application of the Hopf-Friedrichs bifurcation theory", Arch. Rational Mech. Anal. 60 (1976), 371-393.

[29] M. Urabe, "Galerkin's procedure for nonlinear periodic systems", Arch. Ratıonal Mech. Anal. 20 (1965), 120-152.

[30] M. Urabe, Nonlinear autonomous oscillations (Academic Press, New York, 1967).

[31] H. Weber, "Numerical solution of Hopf bifurcation problems", Math. Methods Appl. Sci. 2 (1980), 178-190.

[32] A. H. Wickens, "The dynamic stability of railway vehicle wheelsets and bogies having profiled wheels", Internat. J. Solids and Structures I (1965), 319-341.

[33] A. H. Wickens, "The dynamic stability of a simplified four wheel railway vehicle having profiled wheels", Internat. J. Solids and Structures 1 (1965), 385-406. 